\title{
Two-Dimensional Alignment and Displacement Sensor based on Movable Broadside-coupled Split Ring Resonators
}

\author{
Ali K. Horestani ${ }^{1, *}$, Jordi Naqui ${ }^{2}$, Zahra Shaterian ${ }^{1}$, Derek Abbott ${ }^{1}$, \\ Christophe Fumeaux ${ }^{1}$, and Ferran Martín ${ }^{2}$ \\ ${ }^{1}$ School of Electrical 83 Electronic Engineering, The University of Adelaide, Adelaide, \\ SA 5005, Australia. \\ ${ }^{2}$ GEMMA/CIMITEC, Departament d'Enginyeria Electronica, Universitat Autonoma de \\ Barcelona, 08193 Bellaterra, Spain.
}

\begin{abstract}
This paper proposes a two-dimensional alignment and displacement sensor based on movable broadside-coupled split ring resonators (BC-SRRs). As a basis for this sensor, a one-dimensional displacement sensor based on a microstrip line loaded with BC-SRRs is presented firstly. It is shown that compared to previously published displacement sensors, based on SRR-loaded coplanar waveguides, the proposed one-dimensional sensor benefits from a much wider dynamic range. Secondly, it is shown that with modifications in the geometry of the BC-SRRs, the proposed one-dimensional sensor can be modified and extended by adding a second element to create a high-dynamic range two-dimensional displacement sensor. Since the proposed sensors operate based on a split in the resonance frequency, rather than the resonance depth, they benefit from a high immunity to environmental noise. Furthermore, since the sensors' principle of operation is based on the deviation from symmetry, they are more robust to ambient conditions such as changes in the temperature, and thus they can be used as alignment sensors as well. A prototype of the proposed two-dimensional sensor is fabricated and the concept and simulation results are validated through experiment.
\end{abstract}

Keywords: metamaterials, alignment sensor, displacement sensor, two-dimensional, high dynamic range 


\section{Introduction}

The concept of metamaterials was originally proposed for the realization of artificially engineered bulk materials with positive, near zero, or negative effective permittivity and/or permeability $[1,2,3]$. However, due to their sub-wavelength dimensions, metamaterial fundamental elements such as split ring resonators (SRRs), have also found applications in the design of compact one- and two-dimensional planar circuits such as filters $[4,5,6,7,8,9,10]$, couplers $[11,12,13,14,15]$, and antennas $[16,17,18]$, or to improve the performance of these components $[19,20]$. It has been also shown that due to the high quality factor, subwavelength dimensions, and the localized sensitivity of the resonance to the constituent materials and physical dimensions, SRRs can be used in the design of high sensitivity and high resolution sensors $[21,22,23,24,25,26,27,28]$, or to enhance the sensitivity of the conventional sensors [29].

Recently, one- and two-dimensional displacement sensors based on symmetry properties of SRR-loaded coplanar waveguide (CPW) have been proposed $[21,27,24]$. In these sensors a displacement was characterized by measuring the depth of the notch in the transmission coefficient of the loaded CPW. Other displacement sensors based on the shift of resonance frequency are reported in $[30,31]$. The main advantage of the sensors based on symmetry properties over those based on the shift of resonance frequency is that external (ambient) conditions may affect the resonators' resonance frequency but not the transparency of the loaded lines with perfectly aligned resonators. Thus, the sensors based on symmetry properties of SRR-loaded CPW are more robust and specially suited for alignment purposes. However, they have a fundamental dynamic range limitation, set by the width of the CPW signal strip.

This paper proposes a two-dimensional displacement sensor based on a microstrip line loaded with modified broadside-coupled SRRs (BC-SRRs). In contrast to the above-mentioned displacement sensors [21, 27, 24], in which a fundamental dynamic range limit was dictated by the CPW's lateral dimension, the proposed sensor has virtually no dynamic range limit. Moreover, since the operation principle is based on symmetry properties of the structure, it is more robust to ambient conditions and can be also used as a two-dimensional alignment sensor.

In contrast to other techniques for measuring displacement, such as laserbased displacement sensors, etc, our proposed technique is especially suitable 
for measuring relative displacement between two two-dimensional surfaces, and of special interest for detecting any misalignment between such surfaces.

\section{One-Dimensional Displacement Sensor Based on Broadside Cou- pled Split Ring Resonators}

Figures 1(a) and (b) illustrate the side and top view of the one-dimensional displacement sensor [31], which is composed of a microstrip line loaded with a pair of BC-SRRs. Each BC-SRR is composed of two U-shaped split-rings printed on different layers, on top of each other and open in opposite directions. Note that in this structure one of the rings is printed on the same layer as the microstrip line, so it is fixed, while the second ring is on the top layer of a second substrate, which can be displaced along the direction of the microstrip line, as shown by the red arrows in the figure. The aim of the sensor is to measure this displacement.

The BC-SRR can be modeled as a parallel LC resonator [32], in which the equivalent capacitance corresponds to the capacitance of the overlapping metallic area of the two U-shaped rings, and the equivalent inductance corresponds to the rectangular loop formed by the two U-shaped rings. The BC-SRR's resonance frequency is related to its equivalent capacitance and inductance by $f=1 / 2 \pi \sqrt{L C}$. In the configuration shown in the figure, an increase in the displacement $\Delta x$ of the upper ring results in a decrease in the equivalent capacitance and an increase in the equivalent inductance of the BC-SRR. However, as shown in the simulation results of Fig. 1 (c) and analyzed in Appendix, the change in the equivalent capacitance is dominant, which results in a shift of resonance frequency to higher frequencies. The shift in the resonance frequency can therefore be used to sense the amount of displacement in $x$ direction. Note that the dynamic range of the sensor has virtually no intrinsic geometric limitation and can be increased by using longer BC-SRRs. Dimensions of the simulated structure are listed in the caption of Fig. 1.

Figures 2(a) and (b) illustrate the side and top views of a novel onedimensional sensor, in which the BC-SRRs are rotated such that a lateral displacement increases the equivalent capacitance of one of the BC-SRRs, while it decreases the equivalent capacitance of the other BC-SRR. Also, the equivalent inductance of one of the resonators is decreased, while that of the other resonator is increased. Therefore, as shown with the blue solid line in Fig. 2(c), at the initial position, i.e. for $\Delta y=0 \mathrm{~mm}$, when the 


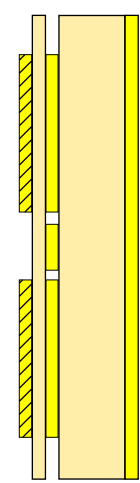

(a)

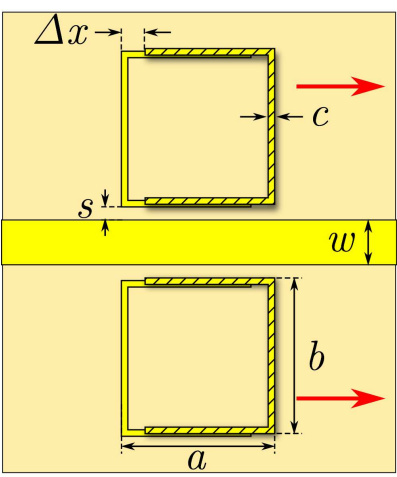

(b)

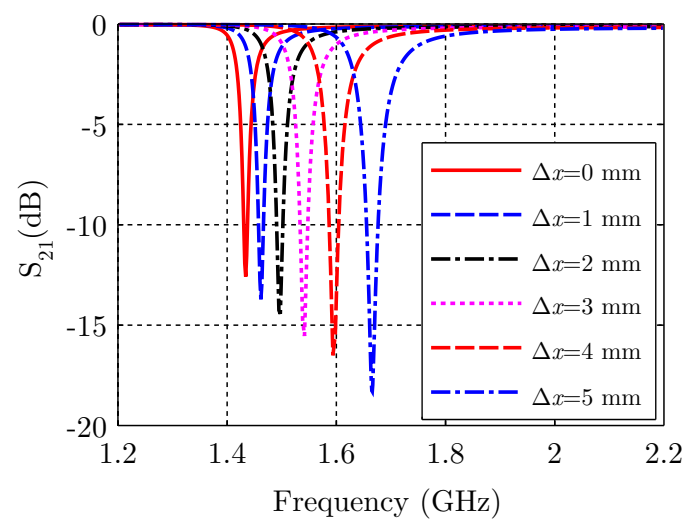

(c)

Figure 1: (a) Side view and (b) top view of the displacement sensor based on BC-SRRs. Rogers $R_{4} 003$ substrates with relative permittivity of 3.38 and copper metallization with thickness of $35 \mu \mathrm{m}$ are used. The thickness of the bottom and top substrates are $1.524 \mathrm{~mm}$ and $0.203 \mathrm{~mm}$, respectively. The width of the microstrip line is $w=3.3 \mathrm{~mm}$ which corresponds to a $50 \Omega$ characteristic impedance. Other dimensions of the structure are $a=10.2 \mathrm{~mm}, b=12.4 \mathrm{~mm}, c=0.4 \mathrm{~mm}$, and $s=0.2 \mathrm{~mm}$. (c) Simulated transmission coefficients of the structure for different values of displacement from $\Delta x=0 \mathrm{~mm}$ to $\Delta x=5 \mathrm{~mm}$ in steps of $1 \mathrm{~mm}$.

symmetry plane of the top substrate is aligned with the symmetry plane of the microstrip line, both resonators are identical, and only one notch in the transmission coefficient of the loaded TL appears at $f_{y 0}$. However, when the symmetry is broken by a lateral displacement of the upper substrate, one of the BC-SRRs becomes smaller than the other one, resulting in the splitting of the resonance in two notches $f_{y 0}$ and $f_{y 1}$ in the transmission spectrum of the TL. Thus, the frequency difference between the two notches $\Delta f_{y} \stackrel{\text { def }}{=} f_{y 1}-f_{y 0}$ can be used to sense the lateral displacement.

Simulation results show that compared to the previously published displacement sensors [33, 27], where an intrinsic dynamic range limitation of about $1 \mathrm{~mm}$ was dictated by the CPW's lateral dimensions, the proposed sensor has a much larger dynamic range of $3 \mathrm{~mm}$. Note that this work is a proof-of-concept, upon which the dynamic range in the proposed sensors can be enhanced by enlarging the resonant elements in the direction of displacement. By doing this, the variable capacitance between intersecting (i.e. face-to-face) metallic elements of the resonator can provide a wider dy- 


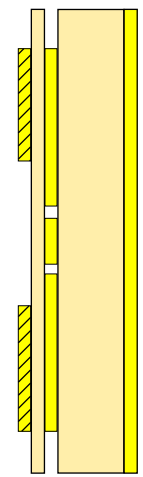

(a)

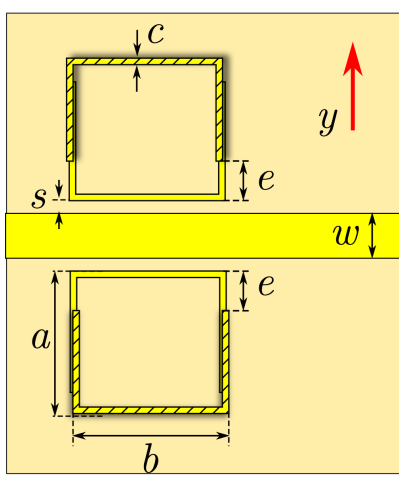

(b)

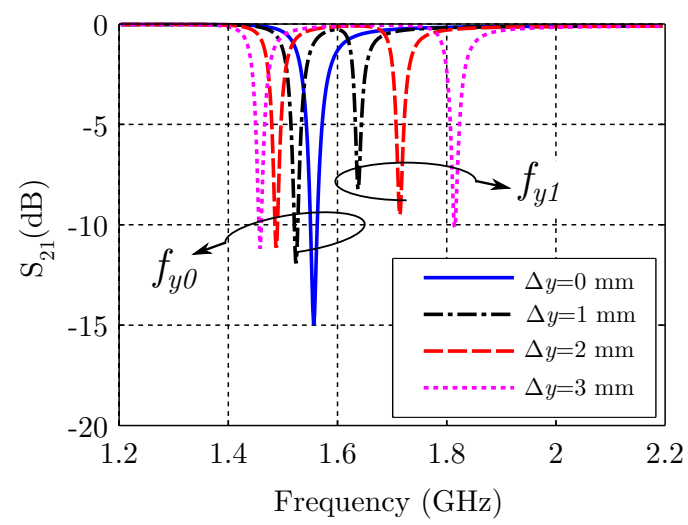

(c)

Figure 2: (a) Side view and (b) top view of the modified one-dimensional displacement sensor. Rogers $R 04003$ substrates with relative permittivity of 3.38 and $35 \mu \mathrm{m}$ thick copper metallization are used. The thickness of the bottom and top substrates are 1.524 $\mathrm{mm}$ and $0.203 \mathrm{~mm}$, respectively. The width of the microstrip line is $w=3.3 \mathrm{~mm}$ which corresponds to a $50 \Omega$ characteristic impedance. Dimensions of the BC-SRRs are as follows: $a=13.2 \mathrm{~mm}, b=12.4 \mathrm{~mm}, c=0.4 \mathrm{~mm}, s=0.2 \mathrm{~mm}$, and $e=3 \mathrm{~mm}$. (c) Simulated transmission coefficients of the structure for different displacements $\Delta y=0 \mathrm{~mm}$ to $\Delta y=$ $5 \mathrm{~mm}$ in steps of $1 \mathrm{~mm}$.

namic range. Also, since the proposed sensor operates based on a split in the resonance frequency rather than the resonance depth, it benefits from a higher immunity to environmental noise [33]. Furthermore, since the operation principle of the sensor is based on the break in symmetry arising from the displacement, the sensor is robust to variable ambient conditions such as changes in the temperature, and can be also used as alignment sensor [24, 27].

\section{Two-Dimensional Displacement Sensor}

With some geometrical modifications, the proposed one-dimensional sensor can be extended to a dual-element configuration operating as a twodimensional alignment and displacement sensor. To this end, as shown in Fig. 3, one possible strategy is to introduce a right angle bend in the microstrip line, and etch a pair of BC-SRRs with orthogonal orientation in each section. The pairs of BC-SRRs need to have different dimensions to resonate at sufficiently different frequencies such that a displacement in $x$ 
direction can be distinguished from that in $y$ direction. Furthermore, the geometry of the resonators needs to be modified so that the resonant frequency is altered by the displacement in one direction only. To this end, the upper rings are replaced with straight strips. In this configuration, provided the strips are long enough, the pair of the modified resonators that are coupled to the horizontal section of the microstrip line is only sensitive to a displacement in $y$ direction, while the pair of the modified BC-SRRs coupled to the vertical section of the line is only responsive to a displacement in $x$ direction. In a practical system, in order to avoid abrasion of metallic layers and substrates, and consequently to increase the repeatability of alignment and displacement sensing, metallic layers are printed face-to-face, and an air gap is added between the top and bottom substrates, as shown in the side view of Fig. 3(a). In order to increase the coupling between the modified BC-SRRs and the microstrip line, the middle section of the U-shaped rings are narrowed [34].

Figure 4(a) and (b) depict the simulated response of the proposed twodimensional sensor to displacements in $x$ and $y$ directions, respectively. Both sets of simulations are conducted by using parameters of Rogers $R 04003$ material with relative permittivity $\epsilon_{r}=3.38$ and thickness of $0.81 \mathrm{~mm}$ for the bottom and top substrates. The two substrates are separated with a $0.25 \mathrm{~mm}$ air gap. The structure's dimensions are listed in the caption of Fig. 3. Figure 4(a) depicts the simulated transmission coefficients of the proposed two-dimensional sensor for different values of displacement in $x$ direction from $0 \mathrm{~mm}$ to $3 \mathrm{~mm}$ in steps of $1 \mathrm{~mm}$ when no displacement in $y$ direction is applied, i.e. $\Delta y=0 \mathrm{~mm}$. At initial position, when both pairs of the modified BC-SRRs are aligned with the symmetry plane of the corresponding sections of the microstrip line, only two notches at $f_{x 0}$ and $f_{y 0}$ appear in the transmission spectrum of the line (blue solid line). The resonance at frequencies $f_{x 0}$ and $f_{y 0}$ are associated with Pair 1 and Pair 2, respectively. However, as shown in the figure, a displacement in $x$ direction results in the splitting of the resonance of the Pair 1 in two notches at $f_{x 0}$ and $f_{x 1}$. The difference between the two frequencies, i.e. $\Delta f_{x} \stackrel{\text { def }}{=} f_{x 1}-f_{x 0}$, is increased with an increase in displacement in $x$ direction, thus it can be used for sensing the value of $\Delta x$. Similarly, Fig. 4(b) shows the simulated transmission coefficients of the proposed sensor for variation of $\Delta y$ from $0 \mathrm{~mm}$ to $3 \mathrm{~mm}$ in steps of $1 \mathrm{~mm}$ while the Pair 1 is aligned with the symmetry plane of the vertical section of the microstrip line, i.e. $\Delta x=0 \mathrm{~mm}$. The figure 


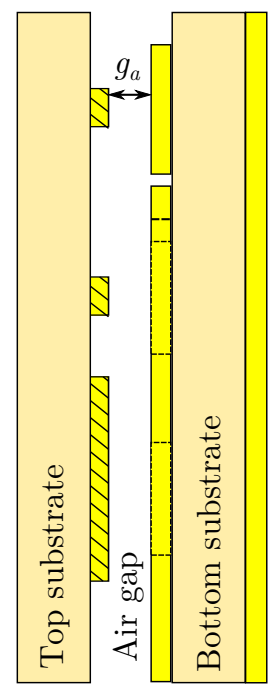

(a)

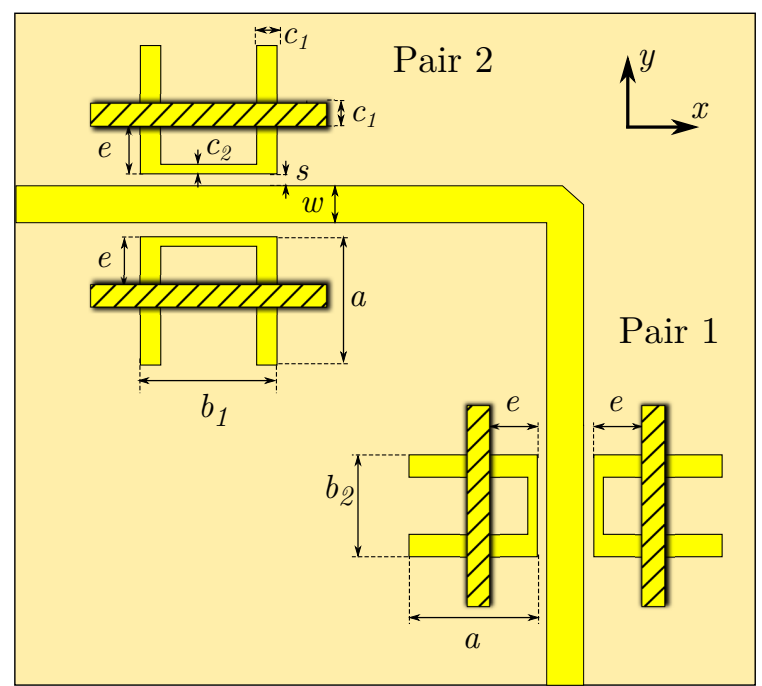

(b)

Figure 3: (a) Side view and (b) top view of the proposed two-dimensional displacement sensor. Rogers $R 04003$ material with relative permittivity $\epsilon_{r}=3.38$ and thickness of $0.81 \mathrm{~mm}$ is used for the bottom and top substrates. The two substrates are separated with a air gap $g_{a}=0.25 \mathrm{~mm}$. The microstrip line has a width $w=1.85 \mathrm{~mm}$, which corresponds to a $50 \Omega$ line. The dimensions of the resonators are as follows: $a=12.2 \mathrm{~mm}$, $b_{1}=15.5 \mathrm{~mm}, b_{2}=10.5 \mathrm{~mm}, c_{1}=2.5 \mathrm{~mm}, c_{2}=1.2 \mathrm{~mm}, s=0.2 \mathrm{~mm}$, and at initial position $e=4.8 \mathrm{~mm}$. 


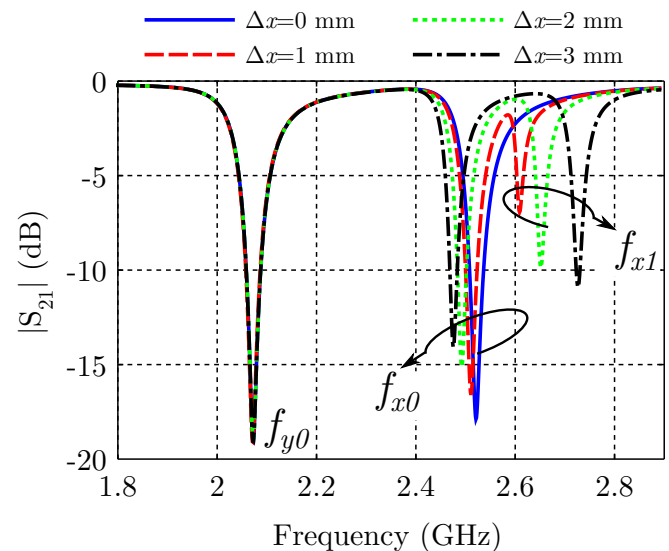

(a)

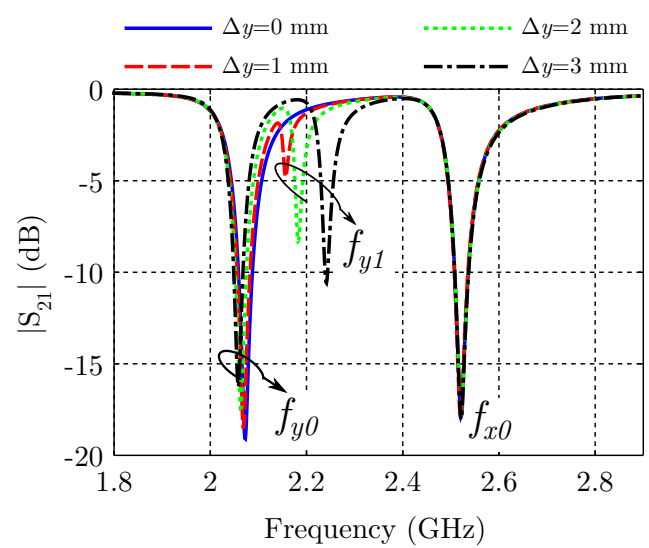

(b)

Figure 4: Simulated transmission coefficients of the proposed sensor (a) for different values of displacement in $x$ direction from $0 \mathrm{~mm}$ to $3 \mathrm{~mm}$ in steps of $1 \mathrm{~mm}$, while $\Delta y=0 \mathrm{~mm}$, and (b) for different values of displacement in $y$ direction from $0 \mathrm{~mm}$ to $3 \mathrm{~mm}$ in steps of $1 \mathrm{~mm}$, while $\Delta x=0 \mathrm{~mm}$.

clearly shows that $\Delta f_{y} \stackrel{\text { def }}{=} f_{y 1}-f_{y 0}$ increases with $\Delta y$, thus it can be used for sensing a displacement in $y$ direction.

With the structure of Fig. 3, the limitation in dynamic range is dictated by the distance where the straight strips and U-shaped resonators do not overlap. Since $e=4.8 \mathrm{~mm}$ at the initial position and considering the width of the metallic strips, the measurable displacement in this case cannot exceed roughly $4 \mathrm{~mm}$. For this reason we have consider displacements up to $3 \mathrm{~mm}$.

\section{Experimental Results}

In order to validate the concept and the simulation results of the previous section, the designed two-dimensional sensor has been fabricated and measured. Dimensions of the prototype corresponds to the simulated structure of the previous section, as given in the caption of Fig. 3. Photographs of the top and bottom substrates of the fabricated prototype are depicted in Figs. 5(a) and (b). Fig. 6 shows a photograph of the measurement setup, which is composed of a pair of micrometer actuators for adjusting the air space between the two substrates, as well as two pairs of micrometer actuators for accurate displacement in $x$ and $y$ directions. In this configuration the 


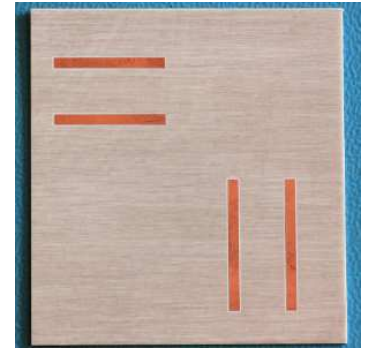

(a)

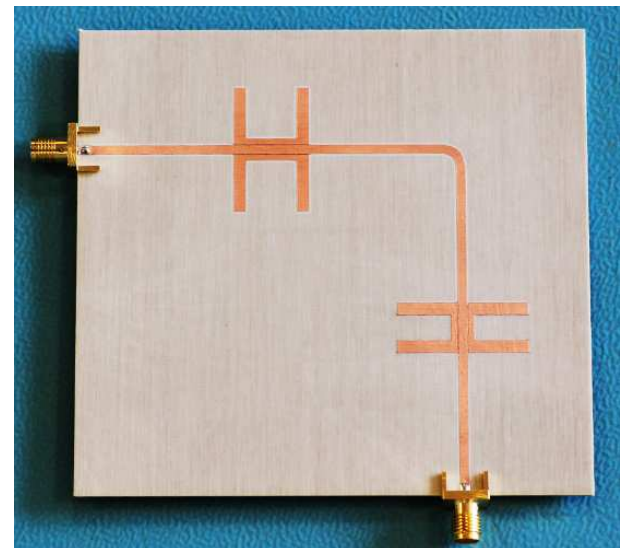

(b)

Figure 5: Photographs of the fabricated prototype (a) top and (b) bottom substrates.

gap uniformity is in principle guaranteed because the considered substrates are of sufficient rigidity in our experiments. In an arbitrary general application, where rigidity may be insufficient it will be a matter of appropriate choice of substrate material to suit the task.

Since the purpose of this paper is to provide an initial proof-of-principle a vector network analyzer, which is not a low-cost instrument, is used for the measurement of the transmission coefficient of the sensor. However, in a practical application, a voltage-controlled oscillator can be used to excite the sensor. By sweeping the frequency in the region of interest, by means of an envelope detector cascaded at the output port, the frequencies where the notch (for alignment) or notches (for misalignment) appear can be detected.

Figure 7(a) depicts the measured transmission coefficients of the sensor for different values of $\Delta x=1 \mathrm{~mm}, 2 \mathrm{~mm}$, and $3 \mathrm{~mm}$, while the sensor has a fixed displacement of $2 \mathrm{~mm}$ in $y$ direction. The figure shows that while frequency difference $\Delta f_{x}$ is increased form $105 \mathrm{MHz}$ to $267 \mathrm{MHz}, \Delta f_{y}$ does not change. Similarly, Fig. 7(b), shows the measured results for a fixed displacement of $0.5 \mathrm{~mm}$ in $x$ direction, resulting in fixed notches at $f_{x 0}=$ $2.5 \mathrm{GHz}$ and $f_{x 1}=2.6 \mathrm{GHz}$, while $\Delta y$ is changed from $1 \mathrm{~mm}$ to $3 \mathrm{~mm}$ in steps of $1 \mathrm{~mm}$, which can be sensed from the increase in $\Delta f_{y}$ from $95 \mathrm{MHz}$ to $210 \mathrm{MHz}$. The experiment shows that each pair of the modified BC-SRRs are exclusively responsive to a displacement either in $x$ or $y$ direction. Thus, the proposed structure can be efficiently used as a two-dimensional alignment 


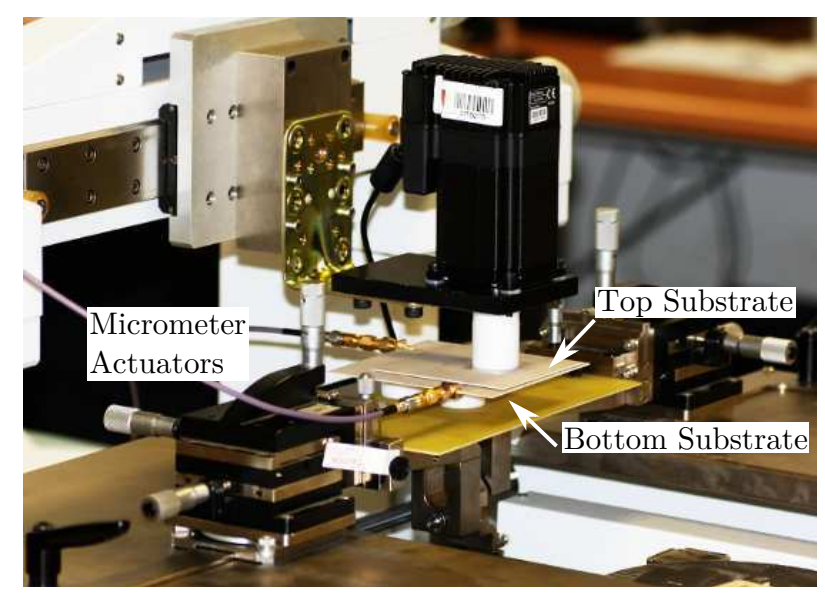

Figure 6: Measurement setup, which is composed of three sets of micrometer actuators for accurate adjustment of the air gap between the substrates as well as displacement in $x$ and $y$ directions.

and displacement sensor. In Figs. 8(a) and (b) the measured $\Delta f_{x}$ and $\Delta f_{y}$ versus displacement in $x$ and $y$ directions, respectively, are compared with those of the simulation results. The satisfactory agreement between the results validates the concept and the simulations. The figure also shows the linearity of the proposed sensor.

Note that, the air gap distance can modify the resonance of the notch, but if there is alignment, only one notch is expected. Thus, for alignment purposes the sensor benefits from robustness to environmental changes. Nevertheless, tolerances in the gap distance may also influence the results. This is actually (along with the fabrication and measurement setup tolerances) the reason for the discrepancy between measurements and simulations in the results of Fig. 8. However, as clearly shown in Fig. 8, the trend of the measured frequency difference is very well matched with the simulated results. This can be quantitatively verified through Pearson's correlation coefficient between simulated and measured data [35]. The correlation coefficient is better than 0.99 for displacement in both $x$ and $y$ directions, which quantitatively verifies the good agreement between the simulated and measured data. Hence, calibration of the sensor can be used in order to remove the effect of fabrication tolerances and the gap distance. Furthermore, since the displacement and alignment sensing in the proposed sensor is based on displacement of the top 


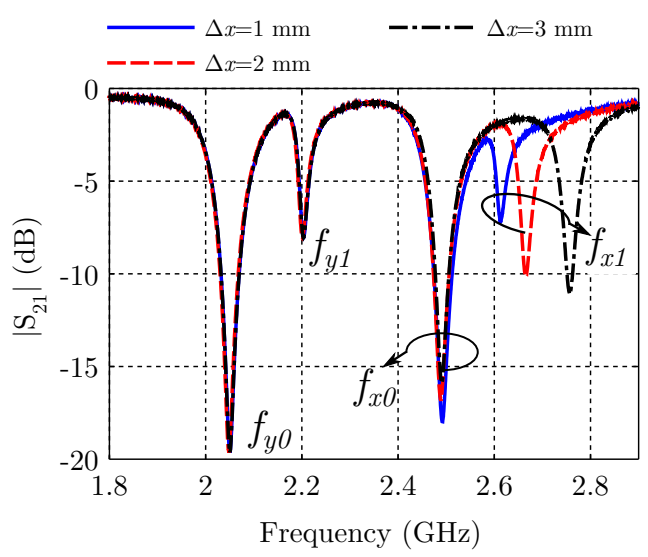

(a)

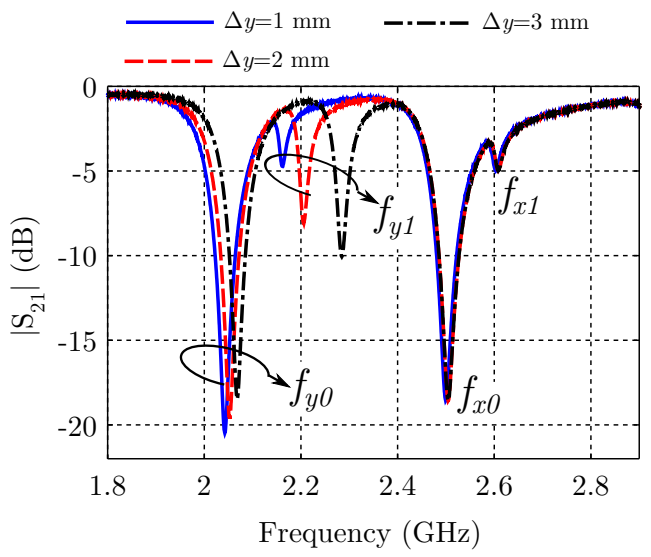

(b)

Figure 7: Measured transmission coefficients (a) for three different values of displacement in $x$ direction, while $\Delta y=2 \mathrm{~mm}$, and (b) for three different values of displacement in $y$ direction, while $\Delta x=0.5 \mathrm{~mm}$.

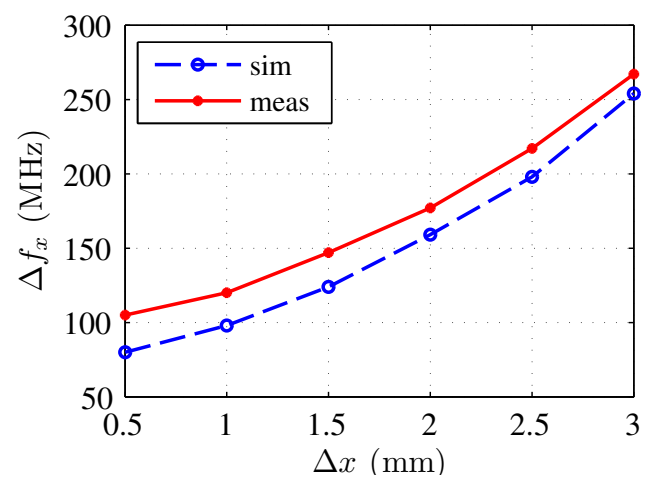

(a)

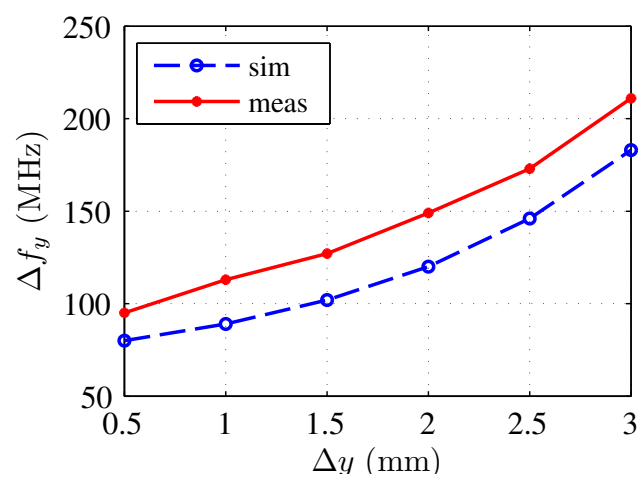

(b)

Figure 8: Comparison between measured and simulated spurious resonance frequency versus displacement: (a) $\Delta f_{x}$ versus $\Delta x$, and (b) $\Delta f_{y}$ versus $\Delta y$. 
substrate without any friction over the bottom substrate, the sensor's functionality benefits from a high repeatability. It is also worth mentioning that one of the main motivations for such a sensor is that it can be easily integrated with MEMS technology, where highly accurate multilayer fabrication technology guarantees the desired gap distance with very good tolerance.

\section{Conclusions}

This work has presented one- and two-dimensional displacement sensors based on microstrip lines loaded with broadside-coupled split-ring resonators. The principle of operation of the proposed sensors is based on the splitting of the resonance frequencies caused by a displacement-induced break of symmetry. It has been shown that compared to the previously published metamaterial-inspired displacement sensors, the proposed sensors benefit from virtually unlimited dynamic range in displacement. Furthermore, since the operation principle of the sensors is based on a split in the resonance frequency, rather than variation in the depth of notch, the sensors are generally immune to the environmental noise. Moreover, because the proposed sensors operate based on the breaking of symmetry, they are also robust to ambient conditions such as changes in the temperature, and can be also used as one- or two-dimensional alignment sensors. The concept and simulation results have been validated through good agreement between the simulated and the experimentally measured response of the a manufactured two-dimensional sensor.

\section{Appendix}

Considering the structure of Fig. 1, the resonators are described by an $L C$ resonant tank coupled to the host transmission line. In a first-order approximation, the resonator exhibits an equivalent inductance given by the whole perimeter of the particle (including both rings), because this is the electric current path. Thus, the equivalent inductance of the particle can be obtained from

$$
L=(2 a+2 b) L^{\prime}+2 x L^{\prime}=2(a+b+x) L^{\prime},
$$

where $L^{\prime}$ is the per-unit length inductance of the resonator, $a$ and $b$ are indicated in Fig. 1, and $x$ is the displacement to be sensed. Notice that an increase in $x$ increases the equivalent inductance. The equivalent capacitance 
of the resonator is due to the broadside capacitance of the face-to-face regions of the individual rings. According to Fig. 1, this capacitance can be written as

$$
C=1 / 2\left[(a-x) C^{\prime}\right]
$$

where $C^{\prime}$ is the per unit length broadside capacitance. Notice that an increase in $x$ decreases the equivalent capacitance of the resonator. The resonance frequency can thus be expressed as

$$
f=\frac{1}{2 \pi \sqrt{L C}}=\frac{1}{2 \pi \sqrt{L^{\prime} C^{\prime}} \sqrt{(a+b+x)(a-x)}},
$$

The derivative of the radicand of the denominator, $R(x)=(a+b+x)(a-x)$, with respect to $x$ is

$$
\frac{d R(x)}{d x}=-(a+b+x)+(a-x)=-b-2 x .
$$

This derivative is negative at $x=0$ and in its vicinity. Hence, the denominator is a decreasing function with $x$, and thus the resonance frequency is an increasing function.

\section{Acknowledgements}

This work has been supported by the Australian Research Council under project DP120100661, and by MINECO (Spain) under projects TEC201017512, CSD2008-00066 and TEC2011-13615-E. Thanks are also given to AGAUR-Generalitat de Catalunya for partially funding this research activity through the project 2009SGR-421. Jordi Naqui is also in debt to MINECO (Spain) for supporting his work through the FPU grant AP2010-0431.

\section{References}

[1] J. B. Pendry, A. Holden, W. Stewart, I. Youngs, Extremely low frequency plasmons in metallic mesostructures, Physical Review Letters 76 (25) (1996) 4773-4776. doi:10.1103/PhysRevLett.76.4773.

[2] J. B. Pendry, A. J. Holden, D. J. Robbins, W. J. Stewart, Magnetism from conductors and enhanced nonlinear phenomena, IEEE Transactions on Microwave Theory and Techniques 47 (11) (1999) 2075-2084. doi:10.1109/22.798002. 
[3] D. R. Smith, W. Padilla, D. Vier, S. Nemat-Nasser, S. Schultz, Composite medium with simultaneously negative permeability and permittivity, Physical Review Letters 84 (18) (2000) 4184-4187. doi:10.1103/PhysRevLett.84.4184.

[4] J. Martel, R. Marqués, F. Falcone, J. D. Baena, F. Medina, F. Martín, M. Sorolla, A new LC series element for compact bandpass filter design, IEEE Microwave and Wireless Components Letters 14 (5) (2004) 210212. doi:10.1109/LMWC.2004.827836.

[5] J. Bonache, I. Gil, J. García-García, F. Martín, Novel microstrip bandpass filters based on complementary split-ring resonators, IEEE Transactions on Microwave Theory and Techniques 54 (1) (2006) 265-271. doi:10.1109/TMTT.2005.861664.

[6] M. Gil, J. Bonache, J. García-García, J. Martel, F. Martín, Composite right/left-handed metamaterial transmission lines based on complementary split-rings resonators and their applications to very wideband and compact filter design, IEEE Transactions on Microwave Theory and Techniques 55 (6) (2007) 1296-1304. doi:10.1109/TMTT.2007.897755.

[7] A. Velez, F. Aznar, J. Bonache, M. D. C. M. Velazquez-Ahumada, J. Martel, F. Martín, A. Vélez, Open complementary split ring resonators (OCSRRs) and their application to wideband CPW band pass filters, IEEE Microwave and Wireless Components Letters 19 (4) (2009) 197-199. doi:10.1109/LMWC.2009.2015490.

[8] A. K. Horestani, Z. Shaterian, W. Withayachumnankul, C. Fumeaux, S. Al-Sarawi, D. Abbott, Compact wideband filter element based on complementary split-ring resonators, in: Proc. SPIE Smart NanoMicro Materials and Devices, Melbourne, Australia, 2011, p. 820431. doi:10.1117/12.902754.

[9] A. K. Horestani, Z. Shaterian, S. Al-Sarawi, D. Abbott, C. Fumeaux, Miniaturized bandpass filter with wide stopband using complementary spiral resonator, in: Proc. Asia-Pacific Microwave Conference (APMC), 2012, pp. 550-552. doi:10.1109/APMC.2012.6421660.

[10] A. K. Horestani, W. Withayachumnankul, A. Chahadih, A. Ghaddar, M. Zehar, D. Abbott, C. Fumeaux, T. Akalin, Metamaterial-inspired 
bandpass filters for terahertz surface waves on Goubau lines, IEEE Transactions on Terahertz Science and Technology 3 (6) (2013) 851858.

[11] C. Caloz, T. Itoh, A novel mixed conventional microstrip and composite right/left-handed backward-wave directional coupler with broadband and tight coupling characteristics, IEEE Microwave and Wireless Components Letters 14 (1) (2004) 31-33. doi:10.1109/LMWC.2003.821506.

[12] A. Lai, C. Caloz, T. Itoh, Composite right/left-handed transmission line metamaterials, IEEE Microwave Magazine 5 (3) (2004) 34-50. doi:10.1109/MMW.2004.1337766.

[13] E. Jarauta, M. A. G. Laso, T. Lopetegi, F. Falcone, M. Beruete, J. D. Baena, A. Marcotegui, J. Bonache, J. García, R. Marqués, F. Martín, Novel microstrip backward coupler with metamaterial cells for fully planar fabrication techniques, Microwave and Optical Technology Letters 48 (6) (2006) 1205-1209. doi:10.1002/mop.21579.

[14] K.-Y. Liu, C. Li, F. Li, A new type of microstrip coupler with complementary split-ring resonator, Microwave and Optical Technology Letters 49 (7) (2007) 1613-1616. doi:10.1002/mop.22522.

[15] Y. Wang, Y. Zhang, F. Liu, L. He, H. Li, H. Chen, C. Caloz, Simplified description of asymmetric right-handed composite right/left-handed coupler in microstrip chip technology, Microwave and Optical Technology Letters 49 (9) (2007) 2063-2068. doi:10.1002/mop.22643.

[16] F. J. Herraiz-Martínez, G. Zamora, F. Paredes, F. Martín, J. Bonache, Multiband printed monopole antennas loaded with OCSRRs for PANs and WLANs, IEEE Antennas and Wireless Propagation Letters 10 (2011) 1528-1531. doi:10.1109/LAWP.2011.2181309.

[17] F. J. Herraiz-Martínez, F. Paredes, G. Zamora, F. Martín, J. Bonache, Dual band printed dipole antenna loaded with open complementary split-ring resonators for wireless applications, IEEE Microwave and Wireless Components Letters 54 (4) (2012) 1014-1017. doi:10.1002/mop.26728.

[18] F. Paredes, G. Zamora, S. Zufanelli, F. J. Herraiz-Martínez, J. Bonache, F. Martín, Recent advances in multiband printed antennas based on 
metamaterial loading, Advances in OptoElectronics 2012 (2012) 1-12. doi:10.1155/2012/968780.

[19] J. García-García, F. Martín, F. Falcone, J. Bonache, J. D. Baena, I. Gil, E. Amat, T. Lopetegi, M. A. G. Laso, J. Iturmendi, M. Sorolla, R. Marqués, Microwave filters with improved stopband based on subwavelength resonators, IEEE Transactions on Microwave Theory and Techniques 53 (6) (2005) 1997-2006. doi:10.1109/TMTT.2005.848828.

[20] S. Shi, W.-W. Choi, W. Che, K.-W. Tam, Q. Xue, Ultra-wideband differential bandpass filter with narrow notched band and improved commonmode suppression by DGS, IEEE Microwave and Wireless Components Letters 22 (4) (2012) 185-187. doi:10.1109/LMWC.2012.2187885.

[21] J. Naqui, M. Durán-Sindreu, F. Martín, Novel sensors based on the symmetry properties of split ring resonators (SRRs)., Sensors 11 (8) (2011) 7545-53. doi:10.3390/s110807545.

[22] J. J. Yang, M. Huang, J. Sun, Double Negative Metamaterial Sensor Based on Microring Resonator, IEEE Sensors Journal 11 (10) (2011) 2254-2259. doi:10.1109/JSEN.2011.2132798.

[23] M. Schueler, C. Mandel, M. Puentes, R. Jakoby, Metamaterial inspired microwave sensors, IEEE Microwave Magazine 13 (2) (2012) 57-68. doi:10.1109/MMM.2011.2181448.

[24] J. Naqui, M. Durán-Sindreu, F. Martín, Alignment and position sensors based on split ring resonators, Sensors 12 (9) (2012) 11790--11797. doi:10.3390/s120911790.

[25] W. Withayachumnankul, K. Jaruwongrungsee, C. Fumeaux, D. Abbott, Metamaterial-inspired multichannel thin-film sensor, IEEE Sensors Journal 12 (5) (2012) 1455-1458. doi:10.1109/JSEN.2011.2173762.

[26] W. Withayachumnankul, K. Jaruwongrungsee, A. Tuantranont, C. Fumeaux, D. Abbott, Metamaterial-based microfluidic sensor for dielectric characterization, Sensors and Actuators A: Physical 189 (2013) 233-237. doi:10.1016/j.sna.2012.10.027. 
[27] A. K. Horestani, C. Fumeaux, S. Al-Sarawi, D. Abbott, Displacement sensor based on diamond-shaped tapered split ring resonator, IEEE Sensors Journal 13 (4) (2013) 1153-1160. doi:10.1109/JSEN.2012.2231065.

[28] A. K. Horestani, D. Abbott, C. Fumeaux, Rotation sensor based on horn-shaped split ring resonator, IEEE Sensors Journal 13 (8) (2013) 3014-3015. doi:10.1109/JSEN.2012.2231065.Copyright.

[29] S. A. Taya, M. M. Shabat, Sensitivity enhancement in optical waveguide sensors using metamaterials, Applied Physics A 103 (3) (2011) 611-614. doi:10.1007/s00339-011-6406-0.

[30] C. Mandel, B. Kubina, Passive chipless wireless sensor for twodimensional displacement measurement, in: 41st European Microwave Conference (EuMC), 2011, pp. 79-82.

[31] Z. Shaterian, A. K. Horestani, C. Fumeaux, Metamaterial-Inspired Displacement Sensor with High Dynamic Range, in: Proc. 4th International Conference on Metamaterials, Photonic Crystals and Plasmonics, META'13, Vol. 1, Sharjah, United Arab Emirates, 2013, pp. 9-11.

[32] R. Marqués, F. Mesa, J. Martel, F. Medina, Comparative analysis of edge- and broadside-coupled split ring resonators for metamaterial design-theory and experiments, IEEE Transactions on Antennas and Propagation 51 (10) (2003) 2572-2581. doi:10.1109/TAP.2003.817562.

[33] J. Naqui, M. Durán-Sindreu, F. Martín, On the symmetry properties of coplanar waveguides loaded with symmetric resonators: Analysis and potential applications, in: MTT-S International Microwave Symposium Digest, IEEE, 2012, pp. 1-3. doi:10.1109/MWSYM.2012.6258390.

[34] A. K. Horestani, C. Fumeaux, S. Al-Sarawi, D. Abbott, Split Ring Resonators With Tapered Strip Width for Wider Bandwidth and Enhanced Resonance, IEEE Microwave and Wireless Components Letters 22 (9) (2012) 450-452. doi:10.1109/LMWC.2012.2211341.

[35] J. L. Rodgers, W. A. Nicewander, Thirteen Ways to Look at the Correlation Coefficient, The American Statistician 42 (1) (1988) 59. doi: $10.2307 / 2685263$. 